\title{
Early stopping turbo iteration at low SNR for CE-based stopping criteria
}

\begin{abstract}
The Cross-Entropy-based (CE-based) stopping criteria advantage is able to terminate early in the high signal-to-noise ratio (SNR) while maintain the bit error rate (BER) performance. Unfortunately, the criteria fails to cope with low SNR region and make the decoder iterates until maximum or infinite iteration. This paper proposed an early termination technique at low SNR for the CE-based stopping criteria using the decoding threshold derived by the measurement of reliability (MOR) at low SNR. In the simulation results and analysis, we compare the average iteration number (AIN) and the bit-error rate (BER) performance between the proposed combination methods with the existing CE-based stopping criteria. From the results, the combination method capable to reduce the AIN at low SNR with minimum one AIN while maintaining the AIN at high SNR as the traditional method. This significant reduction could reduce delay and complexity of existing CE-based stopping criteria while maintaining the BER performances.
\end{abstract}

Keyword: Cross-entropy; Turbo coding; Stopping iteration 